\title{
PROGRESIÓN Y ESCALONAMIENTO EN EL CONSUMO DE DROGAS: EVIDENCIA PARA ARGENTINA ${ }^{\circ \circ}$
}

\author{
DRUG CONSUMPTION PROGRESSION AND STEPPING \\ STONE EFFECT: EVIDENCE FOR ARGENTINA
}

\author{
María Inés Lara* \\ Monserrat Serio* \\ María Noelia Garbero**
}

enviado: 18 diciembre 2018 - aceptado: 10 julio 2019

\begin{abstract}
Resumen
El objetivo de este trabajo es estudiar, para Argentina, la progresión en el consumo de drogas que se inicia con el consumo de drogas legales y avanza hacia el consumo de marihuana, cocaína y otras drogas ilícitas. Utilizando la ENPreCoSP del 2011, se analiza la relación entre el consumo de alcohol y tabaco y su edad de inicio con el consumo de marihuana incluyendo variables de propensión y acceso. A su vez, se estudia la relación entre el consumo de marihuana y su edad de inicio con el consumo de otras drogas. Los resultados obtenidos van en línea con la teoría de escalonamiento, el consumo de drogas legales y su inicio más temprano tienen una relación positiva con la probabilidad de consumir marihuana, y el consumo de esta última también se relaciona directamente con la probabilidad de consumir otras drogas ilícitas.
\end{abstract}

Lara, M. I., Serio, M., \& Garbero, M. M. (2019). Progresión y escalonamiento en el consumo de drogas: evidencia para Argentina. Estudios económicos, 36 (73), 5-42.

- Esta investigación es parte del Proyecto Bienal 2016-2018 "Economía y Comportamientos No Saludables" (06-D212), subsidiado por la SECTYP-UNCuyo de Argentina, Res. 3853/16. Agradecemos los comentarios de dos evaluadores anónimos que han contribuido a enriquecer y mejorar el trabajo. Este artículo cuenta con un anexo digital disponible en la revista en su versión electrónica.

* Universidad Nacional de Cuyo, Facultad de Ciencias Económicas.

Correo electrónico: mariaines.lara@fce.uncu.edu.ar

** Universidad Nacional de Cuyo, Facultad de Ciencias Económicas. Fundación Ideal, Mendoza, Argentina. 
Códigos JEL: I12, D12, D81.

Palabras clave: escalonamiento, puerta de entrada, consumo de drogas, Argentina.

\begin{abstract}
The objective of this paper is to study, for Argentina, the progression in the consumption of drugs that begins with the consumption of legal drugs, such as tobacco and alcohol, and advances to the consumption of marijuana, cocaine, and other illicit drugs. The relation between the consumption of alcohol and tobacco and their age initiation and the probability of consuming marijuana are estimated including propensity and access characteristics. Furthermore, the relationship between marijuana use and its age of onset with the consumption of other drugs is studied. Data from ENPreCoSP of 2011 is used. The results obtained are consistent with the staggering theory. The consumption of legal drugs and their early use have a positive relationship with the probability of consuming marijuana, and also the consumption of marijuana shows the same relationship with the probability of consuming other illicit drugs.
\end{abstract}

JEL Code: I12, D12, D81.

Keywords: stepping stone, gateway, drugs consumption, Argentina. 


\section{INTRODUCCIÓN}

Los modelos basados en teoría de la adicción racional (Becker y Murphy, 1988; Becker et al., 1991; Chaloupka, 1991) consideran que la elección de consumo de sustancias psicoactivas por parte de un individuo es el resultado de un comportamiento racional que tiene en cuenta las consecuencias futuras del consumo pasado y presente en un contexto de maximización intertemporal de utilidad. Esto es, si se observa que un individuo consume drogas, entonces, es posible asumir que la utilidad que deriva de este consumo es superior a los costos percibidos por él.

Sin embargo, desde el punto de vista social, el consumo de sustancias psicoactivas es considerado un problema de política pública (OEA, 2013). En muchos países, la venta de drogas representa una proporción importante de la actividad económica que tiene consecuencias adversas en lo político, lo social y lo económico. Genera corrupción y reducción del poder del Estado, lo que amenaza la gobernabilidad; tiene vínculos con el crimen organizado, aumentando la violencia e incrementando el costo policial y judicial. Estas son algunas consecuencias asociadas a las drogas que se reconocen como las más importantes desde la publicación inicial del trabajo de MacCoun y Reuter (2001).

Por el lado del consumo, las consecuencias negativas incluyen los daños que el uso de drogas ocasiona al consumidor y a terceros. González (2017), siguiendo la metodología propuesta por Nutt et al. (2007) y Nutt (2012), agrupa los daños al usuario en físicos, psicológicos y sociales. En los primeros se encuentran la mortalidad y las enfermedades o daños causados en forma directa o indirecta por el uso de drogas. Los daños psicológicos incluyen dependencia y discapacidad mental. Los daños sociales abarcan la pérdida de bienes físicos y de relaciones interpersonales. Los daños a terceros son agrupados en físicos y sociales. Los primeros abarcan las lesiones, ya sean accidentales o deliberadas. Y los segundos incluyen la actividad delictiva asociada con el financiamiento del hábito y los ilícitos cometidos bajo los efectos del consumo de sustancias psicoactivas; el daño ambiental como consecuencia del uso y producción de drogas; el deterioro de las relaciones familiares; el daño internacional (deforestación, violencia, trata de personas, etc.); el costo económico (reducción de productividad laboral, costos asociados a la recuperación de una adicción o al tratamiento de enfermedades relacionadas); y el daño a la comunidad (efecto sobre la calidad de vida de la comunidad).

En cuanto a las consecuencias en Argentina, según Sedronar (2017), el 18\% de las causas de muertes de las personas entre 15 y 65 años están relacionadas con drogas, directa o indirectamente, lo que implica una tasa bruta de mortalidad por 
droga de 52 personas cada 100000 habitantes. El 74\% de estas defunciones son de hombres, lo que es coherente con el mayor consumo de drogas que presenta el género.

A su vez, en el año 2015 en el país se reportaron 588 homicidios, principalmente de personas entre 20 a 24 años, y un total de 1368 lesiones relacionados con el consumo de drogas (Sedronar, 2017). Si bien, muchos consumidores de drogas probablemente jamás cometan un delito, existe una relación significativa entre las conductas delictivas y el consumo de sustancias psicoactivas. Autores como Goldstein (1985), Parker y Bottomley (1996) argumentan que el uso de drogas conduce a conductas delictivas, mientras que White y Gorman (2000) y Powell (2011) sugieren causalidad inversa. Por otra parte, Dembo et al. (1994) y Stevens et al. (2005) consideran que ambos fenómenos se explican a través de factores comunes.

Adicionalmente a los efectos sobre la salud y el crimen, el consumo de drogas genera un gasto en salud para el sector público y las familias. Si solo se considera el gasto que realiza el gobierno argentino en transferencias y subsidios para tratamientos asistenciales, en el primer trimestre del año 2018 se gastaron 48 millones de pesos (Sedronar, 2018). Esto es una pequeña parte del gasto en salud relacionado con las drogas, ya que no incluye el tratamiento en los hospitales públicos de las enfermedades que son consecuencias del consumo de sustancias psicoactivas ni el gasto de bolsillo de las familias.

De acuerdo con el Informe sobre el consumo de drogas en las Américas 2019 (OEA, 2019), cuánto más temprano se inicie el consumo de drogas, mayores serán los riesgos y daños, por lo que los programas de prevención y otras intervenciones que retrasen la edad de inicio de consumo de drogas deberían recibir alta prioridad.

El objetivo de esta investigación es estudiar, para Argentina, la hipótesis de escalonamiento desde drogas sociales hacia drogas ilegales. Se utiliza la información que brinda la Encuesta Nacional de Prevalencia de Consumo de Sustancias Psicoactivas (ENPreCoSP, INDEC) del 2011. Esta encuesta permite realizar un análisis descriptivo relevante del fenómeno bajo estudio, al mismo tiempo que posibilita estudiar la relación existente entre el consumo y la edad de inicio de las drogas sociales con el consumo de drogas ilegales. Los resultados sugieren que existe una relación positiva entre la condición de fumador y bebedor y sus edades de inicio con la probabilidad de consumir marihuana. A su vez, el consumo previo de marihuana se relaciona positivamente con la probabilidad de progresar hacia el uso de cocaína. 
Diversos estudios empíricos han documentado un patrón común de iniciación al consumo de drogas que comienza con las drogas sociales, tabaco y alcohol, seguido por marihuana y luego por otras drogas ilícitas que se consideran más perniciosas debido a su potencialidad adictiva y a las consecuencias negativas en la salud del consumidor y en la relación con su entorno social y laboral. Este comportamiento secuencial puede ser explicado por efectos causales (es decir, la hipótesis de puerta de entrada) o por características no observables que hacen al individuo más propenso al consumo de drogas (es decir, heterogeneidad no observada).

La hipótesis de puerta de entrada ha influido en la formulación de políticas antidrogas en numerosos países. La misma sostiene que el consumo de una droga incrementa la probabilidad de consumir, en un futuro próximo, otra droga, probablemente más dañina, y esta probabilidad aumenta con la frecuencia de consumo (respuesta a la dosis) (Bretteville-Jensen et al., 2005). Si esto es así, la restricción en el consumo de drogas de inicio (por ejemplo, tabaco o alcohol) redundaría en una disminución de consumo de la subsecuente (por ejemplo, marihuana). Y la legalización o despenalización del consumo de una droga, por ejemplo, marihuana, podría provocar un aumento en el consumo de drogas más peligrosas para la salud del individuo y su entorno (por ejemplo, cocaína y LSD).

Sin embargo, el consumo previo y la correlación son condiciones necesarias, pero no suficientes, para establecer causalidad. La correlación puede reflejar terceros factores que podrían influir en el consumo de varias drogas diferentes, como la propensión individual al consumo y la accesibilidad (Bretteville-Jensen et al., 2005). Las diferencias en estas dos características brindan una explicación alternativa que no fundamenta el comportamiento observado en una relación causal y que tiene implicancias diferentes en lo que respecta al diseño de políticas (Bretteville-Jensen y Jacobi, 2008).

Tal como señalan Fergusson et al. (2006), Bretteville-Jensen y Jacobi (2008), entre otros, los mecanismos que gobiernan la relación entre el consumo de drogas legales y drogas ilícitas aún no se comprenden cabalmente, por lo que las investigaciones empíricas son de suma utilidad. Se necesita un mejor entendimiento de los factores que influyen en el consumo secuencial de drogas para hacer más eficientes las políticas antidrogas. Este trabajo contribuye al análisis de estos patrones de progresión en el consumo en Argentina.

El documento está organizado de la siguiente manera: en la sección 2 se resume la literatura que relaciona el paso del consumo de drogas legales hacia drogas ilícitas. En la sección 3 se describen los datos utilizados en el análisis empírico. 
En la sección 4 se explica la metodología adoptada. Los resultados son expuestos en la sección 5. En la sección 6 se incluye la discusión de las implicancias de política y en la sección 7 se presentan las conclusiones.

\section{ANTECEDENTES}

En muchos países se ha observado un patrón secuencial en la iniciación al consumo de drogas, empezando por las drogas sociales y moviéndose hacia el consumo de drogas ilícitas, generalmente más perjudiciales para la salud. En general, se observa que el consumo de tabaco y alcohol es previo al de marihuana, y este es anterior al de cocaína. La literatura sobre esta temática reconoce a Kandel (1975) como la primera investigadora en observar un patrón sistemático en el uso de sustancias psicoactivas y en desarrollar la teoría de puerta de entrada. De acuerdo con esta autora, es más probable que los consumidores de drogas legales usen subsecuentemente marihuana en relación con aquellos que no las consumen. Esto no implica que todo fumador o consumidor de alcohol consuma marihuana, ni que todos los consumidores de marihuana hayan consumido previamente alguna de las drogas sociales. De manera similar, es más probable que los consumidores de marihuana se inicien posteriormente en el consumo de otras drogas más dañinas que aquellos que no la usan, sin que esto suponga que todos los consumidores de cocaína, éxtasis, entre otras drogas ilegales, hayan consumido previamente marihuana.

Es posible encontrar en la literatura sobre esta temática diferentes justificaciones al efecto puerta de entrada. Pudney (2003) señala tres mecanismos que podrían sentar las bases para un efecto puerta de entrada o escalonamiento en consumo. El primero tiene que ver con la naturaleza de las preferencias del consumidor: el consumo de cierta droga puede crear una necesidad psicológica o fisiológica de experiencias narcóticas más fuertes. Otro mecanismo opera a través de las interacciones sociales: el hecho de obtener y consumir drogas puede poner al consumidor en contacto con proveedores y consumidores de otras drogas controladas con quienes, de otra manera, no se hubiera relacionado. El último mecanismo opera a través de la información y la credibilidad: la no percepción de los efectos dañinos que genera el consumo de drogas legales o que se perciben menos perjudiciales podría dar lugar a una subestimación de los efectos que sobre la salud tienen las drogas más dañinas, por lo que las recomendaciones en contra del uso de sustancias ilícitas se vuelven menos persuasivas.

Bretteville-Jensen et al. (2005) justifican el escalonamiento, argumentando que cuando un individuo consume una droga por primera vez es como si hubiera 
cruzado un umbral, y esta acción hace que sea menos costoso progresar hacia el consumo de otras drogas. En este caso, el efecto causal tendría lugar por una reducción de costos ocasionada por el consumo inicial de drogas en cada etapa. Pacula (1997), asumiendo que el consumo de drogas forma hábitos, propone otra justificación del efecto causal implícito en la hipótesis de puerta de entrada, sugiriendo que el consumo pasado de cualquier droga incrementa la utilidad marginal de consumir dicha droga y cualquier otra.

Otra hipótesis que ha sido sugerida como explicación alternativa de la progresión en el uso de drogas descansa en la existencia de heterogeneidad individual no observada, esto es, terceros factores que influyen simultáneamente sobre el consumo de diferentes sustancias psicoactivas (Bretteville-Jensen et al., 2005). De acuerdo con esta teoría, la relación observada podría no ser causal y ser consecuencia de diferencias en la propensión al consumo de drogas y en la accesibilidad.

La propensión hace referencia a características personales que hacen que el individuo tenga una predisposición o inclinación hacia comportamientos no deseados. Considera que el consumo de drogas es una más de las posibles respuestas conductuales no deseadas ante entornos vulnerables o experiencias personales traumáticas, por lo que no habría un vínculo causal que explique la secuencia observada en consumos, sino que esta correlación respondería a las diferencias en características no observadas de los individuos. Pautassi (2017) señala que el consumo de sustancias psicoactivas involucra factores biológicos, psicológicos y sociales, y factores contextuales - tales como la escasa contención familiar, el estrés, la depresión, la ansiedad, la sensación de soledad, las experiencias traumáticas y la exposición a una cultura que fomenta el consumo de sustancias - que pueden inducir o acelerar el desarrollo de consumo problemático de sustancias. Por ejemplo, Degenhardt et al. (2010) provee evidencia que sugiere que el grado de exposición a drogas es uno de los predictores más importantes del escalonamiento en consumos. Por su parte, Otten et al. (2017) enfatizan la influencia de los amigos, Bretteville-Jensen et al. (2005), Bretteville-Jensen y Jacobi (2008), Melberg et al. (2010), Pacula (1998b) analizan la influencia de entornos familiares y escolares conflictivos.

La accesibilidad se refiere a la habilidad física, a la aceptación cultural, a la legislación sobre drogas prevalecientes y a la asequibilidad (relativa tanto al ingreso del individuo como al precio de las sustancias psicoactivas) que afecta tanto los costos monetarios como los no monetarios del consumo de drogas (BrettevilleJensen et al., 2005; Bretteville-Jensen y Jacobi, 2008). En este sentido, se afirma que las personas pueden comenzar a consumir tabaco y alcohol previo al consumo de marihuana simplemente porque estos son más accesibles. Por ejemplo, para 
analizar accesibilidad, Pacula (1998b) utiliza la edad legal mínima requerida para la compra de alcohol, un índice de criminalidad y una dummy que capta los estados en los que el consumo de marihuana no es considerado un crimen. Bretteville-Jensen y Jacobi (2008) utilizan, además de la respuesta a la pregunta sobre facilidad para obtener una droga determinada, una medida del decomiso de drogas hecho por policía y aduana, el precio de la marihuana y de las anfetaminas, el hecho de vivir en las principales ciudades de Noruega y la prevalencia de consumo de drogas.

Las estimaciones de demandas realizadas por Saffer y Chaloupka (1999) brindan evidencia consistente de complementariedad en el consumo de marihuana, cocaína y heroína, pero sustitubilidad entre alcohol y marihuana. Farrelly et al. (2001), utilizando modelos de datos de panel, encuentran una relación de complementariedad entre tabaco y marihuana, al igual que Pacula (1998a,b), pero además, concluyen que el incremento en las multas por posesión de marihuana y en la probabilidad de arresto disminuyen la probabilidad de consumo de esta droga en jóvenes pero tendrían un efecto leve sobre la frecuencia de consumo ${ }^{1}$.

La investigación realizada por van Ours (2003) concluye que existe una relación positiva entre el consumo de marihuana y cocaína, a pesar de que el efecto puerta de entrada se reduce considerablemente cuando se controla por heterogeneidad no observada. A estos resultados llega estimando modelos de duración bivariados con datos para Amsterdam.

Pudney (2003) utiliza un enfoque similar al de van Ours (2003) con información de Inglaterra y una especificación paramétrica para la heterogeneidad individual no observada, y concluye que los efectos puerta de entrada entre drogas ilícitas se reducen considerablemente y son pequeños. Utilizando modelos probit multivariados e información para Oslo, Bretteville-Jensen et al. (2005) obtienen resultados similares a los de van Ours (2003) y Pudney (2003). Reportan la existencia de efectos puerta de entrada en el uso de drogas legales hacia drogas ilícitas, y que estos efectos se reducen sustancialmente, aunque no desaparecen, si las estimaciones tienen en cuenta la heterogeneidad no observada. Estos autores encuentran que el alcohol es la puerta de entrada hacia el consumo de marihuana, la marihuana hacia las anfetaminas y las anfetaminas hacia la cocaína.

Fergusson et al. (2006) estudian la asociación entre el consumo de marihuana y el uso de otras drogas ilícitas. Emplean información para Nueva Zelanda

1 Tanto Saffer y Chaloupka (1999) como Farrelly et al. (2001) utilizan la National Household Survey on Drug Abuse (NHSDA) de Estados Unidos. 
y proponen un modelo de efectos fijos. Encuentran evidencia que sostiene que el uso frecuente de marihuana está asociado con el incremento en el uso, abuso, dependencia y diversidad en el uso de otras drogas ilícitas (éxtasis, metanfetaminas, cocaína, heroína, morfina, entre otras), aun luego de controlar por características observadas y no observadas. Y la fortaleza de las asociaciones entre marihuana y otras drogas más duras es más fuerte en la adolescencia y disminuye considerablemente con la edad.

Bretteville-Jensen y Jacobi (2008) estudian la influencia del consumo previo de marihuana, la propensión y la accesibilidad en la probabilidad de consumo de drogas ilícitas más fuertes (anfetamina, cocaína, éxtasis y heroína) con información de una muestra representativa de adultos jóvenes noruegos. Proponen una estimación bayesiana de modelos probit bivariados y encuentran que los tres factores importan, aunque domina el primero y le sigue en importancia el tercero.

Melberg et al. (2010) sostienen que la heterogeneidad no observada es crucial para entender la secuencia temporal en el consumo de drogas, por lo que proponen un modelo de hazard latente bivariado para verificar la hipótesis de puerta de entrada. Sus resultados evidencian que el efecto puerta de entrada es estadísticamente significativo y fuerte para un subgrupo de jóvenes problemáticos de Oslo. Dentro de este grupo, el riesgo de progresar en el consumo de drogas duras se duplica si previamente consumieron marihuana ${ }^{2}$. Sin embargo, para el resto de los jóvenes, la relación, si bien sigue siendo significativa, es más débil.

Deza (2015) estima un modelo dinámico de elección discreta a partir de información para Estados Unidos y encuentra un efecto puerta de entrada modesto desde el alcohol hacia el uso posterior de marihuana y otras drogas ilícitas, y desde la marihuana hacia el consumo futuro de drogas más dañinas, y este efecto disminuye con la edad. Adicionalmente, encuentra evidencia de que ambos tipos de drogas son complementarias en utilidad, esto es, consumir simultáneamente alcohol y marihuana produce más utilidad que la suma de las utilidades derivadas de consumir solo alcohol y solo marihuana.

Richmond-Rakerd et al. (2017) proponen modelos de crecimiento latente para analizar el grado en que la edad de inicio de consumo de drogas modera la magnitud de las asociaciones entre consumos futuros de sustancias psicoactivas. Sus resultados indican que las edades de inicio al consumo de alcohol, tabaco y

2 Estos autores agrupan, bajo el concepto de drogas duras, el consumo de anfetamina, cocaína y heroína. 
marihuana están moderadamente correlacionadas entre sí, corroborando la secuencia en la cual los individuos progresan desde el consumo de alcohol y tabaco hacia el consumo de marihuana y otras drogas ilícitas.

Otten et al. (2017) concluyen que la disponibilidad y selección de amigos que consumen sustancias contribuye parcialmente a la progresión, desde la adolescencia hacia la adultez, en el consumo de drogas ilícitas potencialmente más gratificantes y perjudiciales.

Degenhardt et al. (2010) es uno de los pocos trabajos que evalúa la teoría de puerta de entrada para un conjunto grande de países. Estos autores analizan el uso de drogas en 17 países alrededor del mundo con el objetivo de evaluar en qué medida la iniciación al consumo de drogas sigue un patrón consistente entre países. Sus resultados ponen en evidencia que cuando la accesibilidad y/o los factores actitudinales juegan algún papel en el orden en que se inicia el consumo de sustancias, la sustancia que oficia de puerta de entrada puede diferir entre países, dependiendo de las tasas de prevalencias de esa sustancia en relación con otras. Encuentran que el consumo de alcohol y tabaco estaría fuertemente asociado con el consumo posterior de drogas en países en los que el consumo de estas drogas sociales es alto, mientras que la iniciación al consumo de marihuana estaría fuertemente asociada con el subsecuente consumo o uso de otras drogas ilícitas en países con menores tasas de consumo de tabaco y alcohol.

Los investigadores han abordado ambas hipótesis de diferentes maneras, con diferentes métodos econométricos y diferentes fuentes de información y, sin embargo, no hay evidencia contundente que permita inclinarse por una $u$ otra explicación. Más aún, para la literatura de esta temática no ha sido tarea sencilla encontrar efectos causales debido al problema de identificación dada la influencia de ciertas características de los individuos que los hace susceptibles al consumo de drogas y/o las hace más accesibles (heterogeneidad no observada) y a la falta de estrategias empíricas acordes (experimentos naturales). Algunos de los trabajos que incursionan en metodologías que permiten interpretar efectos causales son los de Pacula (1998a,b), DeSimone (1998) y Beenstock y Rahav (2002).

Pacula (1998a,b) analiza la relación entre el consumo de alcohol y el de marihuana a partir de la estimación de demandas individuales utilizando variables instrumentales (VI) para controlar por heterogeneidad no observada. Encuentra que los cambios en el precio de la cerveza tienen un efecto negativo y significativo sobre el consumo de marihuana, implicando que ambas sustancias son complementarias en el consumo. Con la misma fuente de información pero a partir de un 
modelo Tobit estimado por VI, Pacula (1998a) confirma la existencia de una fuerte relación de complementariedad, y además encuentra evidencia de efecto puerta de entrada, dado que el consumo previo de alcohol y tabaco incrementa la probabilidad de usar marihuana. La principal implicación de estos resultados es que las políticas públicas asociadas al control del consumo de alcohol y tabaco serían efectivas para reducir la demanda de marihuana. DeSimone (1998) emplea un enfoque similar al de Pacula (1998b) para analizar la relación entre marihuana y cocaína, y sus resultados evidencian efectos puerta de entrada, esto es, el consumo previo de marihuana incrementa la probabilidad de consumir posteriormente cocaína ${ }^{3}$.

Beenstock y Rahav (2002) prueban el efecto causal puerta de entrada utilizando datos para Israel en el contexto de un experimento natural y con diferentes estrategias empíricas, estimando modelos logit en dos etapas, probit bivariados y modelos de duración. Estos autores encuentran evidencia que indica que el consumo de cigarrillos causa el consumo de marihuana, pero el uso de esta última no tiene un efecto causal sobre el consumo de drogas más fuertes.

También existen antecedentes que evidencian patrones atípicos que violarían la hipótesis de progresión secuencial tradicional (primero consumo de drogas sociales, como alcohol y tabaco, y, luego, progresión hacia otras drogas). Golub y Johnson (1994) y Mackesy-Amiti et al. (1997) analizan estos comportamientos en los casos de consumidores severos de drogas de Estados Unidos. Los autores muestran que la mayoría de este tipo de consumidores no presentan el patrón habitual de progresión de consumo, ya que una proporción significativa empiezan a consumir marihuana antes de haber consumido alcohol por primera vez, y algunos comienzan a consumir otras drogas ilícitas antes de consumir alcohol o marihuana. Por su parte, Aaron Ginzler et al. (2003) analiza patrones atípicos entre jóvenes sin hogar (homeless) de Estados Unidos encontrando que no existe una clara relación entre el consumo de droga de iniciación y el consumo de droga actual. Más aún, encuentran en algunos casos que el consumo de una droga no lleva a una progresión al consumo de otras drogas. En los anteriores trabajos mencionados los consumidores provienen de contextos muy desfavorables o son severos consumidores de droga.

Degenhardt et al. (2009) analizan el rol de trastornos mentales preexistentes y datos sociodemográficos para explicar las violaciones a los patrones típicos de consumo. Concluyen que el uso y la iniciación de consumo de drogas están claramente anidados dentro de un contexto normativo social y la desviación del patrón

3 Pacula (1998a), Pacula (1998b) y DeSimone (1998) utilizan para sus estimaciones la National Longitudinal Survey of Youth (NLSY) de Estados Unidos. 
de consumo parecería responder a trastornos mentales preexistentes, los cuales son factores de riesgo más poderosos para la posterior dependencia de sustancias. Sugieren orientar los esfuerzos de intervención directamente en la detección de estos factores (es decir, trastornos de la internalización, uso de sustancias de inicio temprano) que detectar violaciones de progresión de consumo.

Sartor et al. (2013) explican que, si bien el inicio del consumo de cannabis por lo general sigue al consumo de alcohol, el orden inverso también ocurre y es más común en los afroamericanos (AA) que en los americanos europeos (EE). En sus resultados encuentran que las mujeres AA son tres veces más proclives que las mujeres EE de iniciar el consumo de cannabis antes del consumo de alcohol. De esta manera concluyen que los programas de prevención deben adaptarse a los distintos patrones de consumo de cannabis y las contribuciones relativas de los factores de riesgo al desarrollo de problemas relacionados con el cannabis en diferentes grupos étnicos.

Badiani et al. (2015) utilizan datos de una cohorte longitudinal y encuentran que sus resultados apoyan las hipótesis de "gateway" y "gateway inversa". Esto sugeriría una retroalimentación recíproca que involucra la causalidad simultánea entre el consumo de tabaco y el consumo de marihuana.

Fairman et al. (2019) explican que en los últimos años el incremento de uso de marihuana como iniciación en el consumo de drogas está más asociado a características socioedemográficas como etnia, género, edad y que los patrones de consumo en la actualidad tienen menos poder predictivo. Sartor et al. (2019) consideran patrones típicos aquellos donde se consume alcohol primero y luego marihuana y atípicos aquellos que incluye el consumo de marihuana y alcohol a la misma edad, primero la marihuana, solo marihuana y solo el alcohol. Sus resultados destacan la necesidad de que los programas de prevención consideren la heterogeneidad en los patrones de inicio de consumo.

Hasta donde se tiene conocimiento, no hay investigaciones económicas que hayan estudiado la progresión en el consumo de drogas a partir de datos de Argentina. Por esta razón, se considera que este trabajo contribuye al diagnóstico y entendimiento del escalonamiento observado en el consumo de drogas en el país. 


\section{MARCO LEGAL}

En este apartado se presenta una síntesis de los aspectos más relevantes del marco regulatorio del consumo de sustancias psicoactivas que contribuye a la contextualización del trabajo. No obstante, el análisis y la discusión de estos aspectos exceden los objetivos de la investigación.

En Argentina, el consumo de alcohol está regulado por la Ley 24449 de 1997 de Lucha contra el Alcoholismo. Esta norma prohíbe la venta de bebidas alcohólicas a menores de 18 años; el consumo de bebidas alcohólicas en la vía pública y en el interior de los estadios u otros sitios cuando se realicen en forma masiva actividades deportivas, educativas, culturales y/o artísticas, excepto que lo habilite expresamente la autoridad competente; la publicidad dirigida a menores de 18 años, entre otras medidas.

La Ley 26687 de 2011 de Control de Tabaco contempla las recomendaciones del Convenio Marco para el Control del Tabaco de la Organización Mundial de la Salud. Al igual que la Ley 24449 prohíbe la venta a menores de 18 años, fumar en espacios cerrados de uso público, la publicidad, promoción y patrocinio de cigarrillos o productos elaborados con tabaco, la venta de cigarrillos en centros de enseñanza, establecimientos hospitalarios, edificios públicos, medios de transporte, museos, clubes o lugares de espectáculos públicos; y exige la inclusión de advertencias sanitarias. No obstante, antes del 2011, varias provincias ya habían sancionado normas en el ámbito de su competencia que suplía el déficit de una legislación nacional de control de tabaco.

Ambas normas buscan disminuir los costos sociales del consumo de estas sustancias y ninguna de las dos penaliza directamente al consumidor.

Distinto es el caso de la Ley 23737 de 1989 sobre Tenencia y Tráfico de Estupefacientes. Esta norma está inspirada en las convenciones y tratados internacionales que rigen el control de las sustancias psicoactivas a nivel mundial y tipifica como ilícitos penales las conductas relativas al uso recreacional y personal de estupefacientes, y las reprime con una pena privativa de la libertad. Y si bien la Corte Suprema resolvió, en el 2009, que la tenencia personal no podía ser considerada delito dado el derecho a la privacidad que otorga la Constitución Nacional (Fallo Arriola), la ley no ha sido modificada, por lo que habría una contradicción jurídica (Mansilla, 2017). 
Las políticas fundadas en el prohibicionismo y en la criminalización del consumo no han producido los resultados esperados (Gómez, 2013; UNGASS, 2016; Mansilla, 2017; Samper-Pizano, 2017), lo que ha incentivado a varios países a hacer una interpretación menos restrictiva de los acuerdos internacionales. Muchos de ellos se han volcado hacia una estrategia de abordaje del problema del consumo de drogas denominada "reducción de daños", que tiene como objetivo disminuir las conductas de riesgo y los daños asociados al consumo, tanto para el individuo como para terceros (Mansilla, 2017), la despenalización, la descriminalización, y la regulación selectiva.

La Sesión Especial de la Asamblea General de las Naciones Unidas (UNGASS, 2016) representó una oportunidad para actualizar la estrategia de lucha contra las drogas orientándola hacia la Salud Pública y los Derechos Humanos. No obstante, el prohibicionismo sigue teniendo una fuerte presencia en las políticas públicas de drogas.

\section{DATOS}

Los datos empleados provienen de la Encuesta Nacional de Prevalencia de Consumo de Sustancias Psicoactivas (ENPreCoSP) de Argentina realizada por el INDEC en el año $2011^{4}$. El uso de esta encuesta provee tres ventajas. En primer lugar, brinda información relacionada con el consumo de sustancias psicoactivas y su accesibilidad de una muestra aleatoria de alrededor de 34300 individuos de entre 16 y 65 años de edad, permitiendo trabajar con microdatos. En segundo lugar, la encuesta cuenta con información socioeconómica (edad, género, educación, ingreso, salud, características de la vivienda, entre otras variables) lo que posibilita complementar el estudio. En tercer lugar, tiene representatividad nacional, regional y provincial. De esta manera, la información que brinda la ENPreCoSP permite estudiar la relación entre el consumo de drogas legales, tales como el alcohol y el cigarrillo, y su edad de inicio con el consumo de marihuana y, a su vez, estudiar el escalonamiento del consumo de marihuana hacia consumo de cocaína y éxtasis. No obstante, al tratarse de información autorreportada por consumidores y no consumidores, puede presentar una subdeclaración de consumos de drogas ilegales.

4 Existe una encuesta realizada en el año 2016 pero los datos se encuentran en procesamiento y no están disponibles para el público en general. 
PROGRESIÓN Y ESCALONAMIENTO EN EL CONSUMO DE DROGAS:...

Tabla 1. Consumo de sustancias psicoactivas por género. Argentina

\begin{tabular}{|c|c|c|c|}
\hline Variable & Total & Mujer & Hombre \\
\hline Fumador & 28.93 & 24.03 & 33.94 \\
\hline Fumador severo & 27.43 & 21.13 & 32.01 \\
\hline Exfumador & 23.91 & 22.37 & 25.47 \\
\hline No fumador & 47.12 & 53.54 & 40.56 \\
\hline Edad inicio consumo tabaco & 16.80 & 17.42 & 16.30 \\
\hline Número de cigarrillos por día & 11.50 & 9.92 & 12.66 \\
\hline Consumo de alcohol & 50.70 & 38.84 & 62.79 \\
\hline Edad inicio consumo de alcohol & 17.58 & 18.38 & 16.89 \\
\hline Consumo excesivo de alcohol & 19.07 & 9.28 & 25.24 \\
\hline Consumo de marihuana & 10.67 & 6.87 & 14.55 \\
\hline Edad inicio consumo de marihuana & 19.27 & 20.25 & 18.81 \\
\hline Consumo excesivo de marihuana & 23.67 & 17.30 & 26.07 \\
\hline Consumo de cocaína & 3.09 & 1.06 & 5.16 \\
\hline Edad inicio consumo de cocaína & 19.25 & 19.31 & 19.24 \\
\hline Consumo excesivo de cocaína & 51.77 & 33.98 & 53.92 \\
\hline Consumo de éxtasis & 0.87 & 0.46 & 1.28 \\
\hline Edad inicio consumo de éxtasis & 22.57 & 21.54 & 22.95 \\
\hline Consumo excesivo de éxtasis & 8.70 & 0.00 & 14.27 \\
\hline Consumo de drogas ilegales totales & 11.05 & 7.05 & 15.14 \\
\hline
\end{tabular}

Nota: Valores no condicionados.

Fuente: elaboración propia sobre la base de ENPreCoSP. 
El 49,5\% de los individuos encuestados son hombres, la edad promedio es de 37 años, el $58 \%$ ha terminado algún nivel educativo y solo el $2 \%$ habita en viviendas precarias. Más del $80 \%$ de la muestra declara gozar de un estado de salud bueno o muy bueno.

En relación con el consumo de sustancias psicoactivas, el 29\% fuma y lo ha hecho en los últimos 30 días previos a la encuesta, el 24\% es exfumador (lleva sin fumar más de 30 días) y el $47 \%$ nunca ha probado un cigarrillo. A su vez, el $27 \%$ de los fumadores puede considerarse como fumador severo, es decir aquella persona que fuma más de 15 cigarrillos por día. La edad de inicio promedio de los fumadores es de casi 17 años. Por otra parte, el 50.7\% declara consumir alcohol habiendo iniciado su consumo pasado los 17 años y el 19\% tiene un consumo excesivo de alcohol ${ }^{5}$.

La tasa de prevalencia de consumo de marihuana es del 10.7\%, el 3\% de la población ha consumido cocaína y solo $0.9 \%$ consumió éxtasis. En conjunto, el $11 \%$ de la población consume o consumió alguna droga ilegal. La edad promedio de inicio de consumo de marihuana y cocaína es de 19 años mientras que el éxtasis tiene una edad promedio de inicio de casi 23 años. A su vez, un $24 \%$ de las personas que consumen marihuana declara haber consumido en alguna ocasión más de lo pensado, el 52\% de los consumidores de cocaína considera haber tenido algún consumo excesivo de la sustancia mientras que en el caso de éxtasis el $9 \%$ considera haberse excedido en su consumo alguna vez. En cuanto a las diferencias por género, los hombres presentan mayores tasas que las mujeres en el consumo de drogas legales e ilegales y comienzan a consumir a una edad más temprana.

La tabla 2 presenta el porcentaje de consumidores de la muestra según las siguientes categorías: 1) consumidores que solo han consumido alcohol y/o tabaco; 2) patrón secuencial: consumidores que han consumido alcohol y/o tabaco primero, luego solo marihuana; 3 ) patrón secuencial: consumidores que han consumido alcohol y/o tabaco primero, luego marihuana y, luego, otras drogas; 4) consumidores que solo han consumido marihuana; 5) consumidores que solo han consumido otras drogas; 6) que han consumido marihuana antes que alcohol y tabaco; 7) que han consumido otras drogas ilícitas antes que alcohol y tabaco; 8) que han consumido otras drogas ilícitas antes que marihuana; 9) que no han probado ninguna de las

5 Se sigue la definición de INDEC que considera consumo excesivo cuando el individuo ha consumido, al menos una vez en los últimos 30 días, 8 o más tragos de cerveza y/o 5 tragos de vino y/o 3 tragos bebidas fuertes, ya sea entre semana o en el fin de semana. Para marihuana, cocaína y éxtasis, el consumo excesivo es reportado por el individuo. 
drogas anteriores. Los perfiles de patrones secuenciales mostrarían la importancia relativa del escalonamiento desde drogas sociales hacia marihuana, siendo esta la primera droga ilegal, considerada por varios autores como la droga de entrada hacia consumos más problemáticos (Bretteville-Jensen et al., 2005).

El 72\% de la muestra declara haber consumido alcohol y/o tabaco únicamente, el $5.5 \%$ de la muestra declara algún patrón de consumo secuencial. El $4.5 \%$ reporta haber consumido solamente marihuana luego de haberse iniciado en el consumo de drogas sociales, mientras que solo el 1\% reporta haber primero consumido drogas sociales, luego marihuana para finalmente escalar al consumo de otras drogas. Alrededor del 19\% reporta no haber consumido ningún tipo de drogas.

Tabla 2. Consumidores según patrones secuenciales típicos y atípicos

\begin{tabular}{|c|c|c|c|}
\hline & Obs. & $\begin{array}{l}\text { Obs. } \\
\text { ponderadas }\end{array}$ & $\%$ \\
\hline Solo han consumido alcohol y/o tabaco & 24755 & 16091215 & $72.1 \%$ \\
\hline $\begin{array}{l}\text { Patrones secuenciales } \\
\text { Consumido alcohol y/o tabaco primero, } \\
\text { luego solo marihuana }\end{array}$ & 1532 & 1442349 & $4.5 \%$ \\
\hline $\begin{array}{l}\text { Consumido alcohol y/o tabaco primero, } \\
\text { luego marihuana y, luego, otras drogas. }\end{array}$ & 355 & 352848 & $1 \%$ \\
\hline $\begin{array}{l}\text { Patrones atípicos } \\
\text { Solo han consumido marihuana. }\end{array}$ & 24 & 16200 & $0.1 \%$ \\
\hline Solo han consumido otras drogas. & 2 & 417 & $0 \%$ \\
\hline $\begin{array}{l}\text { Han consumido marihuana antes que } \\
\text { alcohol y tabaco. }\end{array}$ & 77 & 76774 & $0.2 \%$ \\
\hline $\begin{array}{l}\text { Han consumido otras drogas ilícitas antes } \\
\text { que alcohol y tabaco. }\end{array}$ & 33 & 23920 & $0.1 \%$ \\
\hline $\begin{array}{l}\text { Han consumido otras drogas ilícitas antes } \\
\text { que marihuana. }\end{array}$ & 93 & 85149 & $0.3 \%$ \\
\hline $\begin{array}{l}\text { No han probado ninguna de las drogas } \\
\text { anteriores }\end{array}$ & 6448 & 3691384 & $18.8 \%$ \\
\hline
\end{tabular}

Fuente: elaboración propia en base a ENPreCoSP. 
Respecto a los patrones atípicos, los resultados indican que en la muestra a trabajar los mismos son modestos. El porcentaje de consumidores con patrones atípicos alcanza el $0.7 \%$. Solo el $0.1 \%$ reporta haber consumido directamente marihuana y el $0.2 \%$ haber consumido marihuana antes que alguna droga social.

Además, en la figura 1 se observa gráficamente que las funciones de densidad de las edades de inicio de consumo de drogas legales se encuentran más a la izquierda que las funciones de las edades medias de inicio de consumo de drogas ilegales. A su vez, se advierte que las medias de las edades de inicio de consumo de drogas legales son inferiores a las edades medias de inicio de consumo de drogas ilegales. Asimismo, la edad de inicio de consumo de marihuana promedio es previa a la edad de inicio promedio de consumo de éxtasis. Cuando se diferencia por género, se encuentra que, en promedio, los hombres consumen marihuana a una edad más temprana que cocaína.

Figura 1. Función de densidad de la edad de inicio de consumo de sustancias psicoactivas. Argentina 2011

Alcohol, tabaco y marihuana

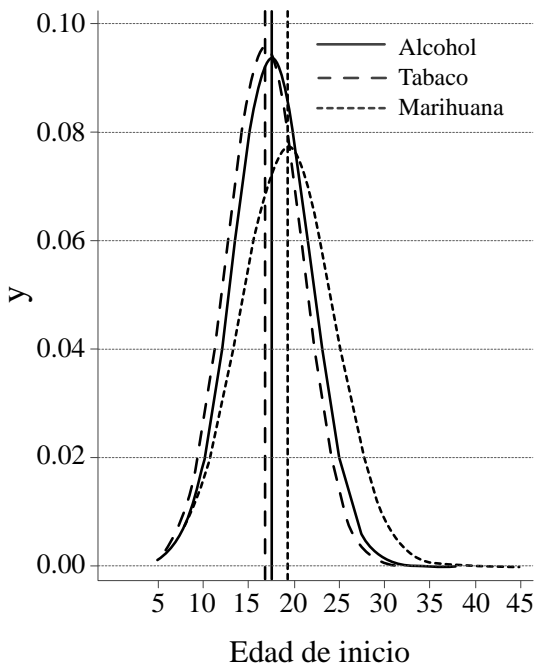

Marihuana, cocaína y éxtasis

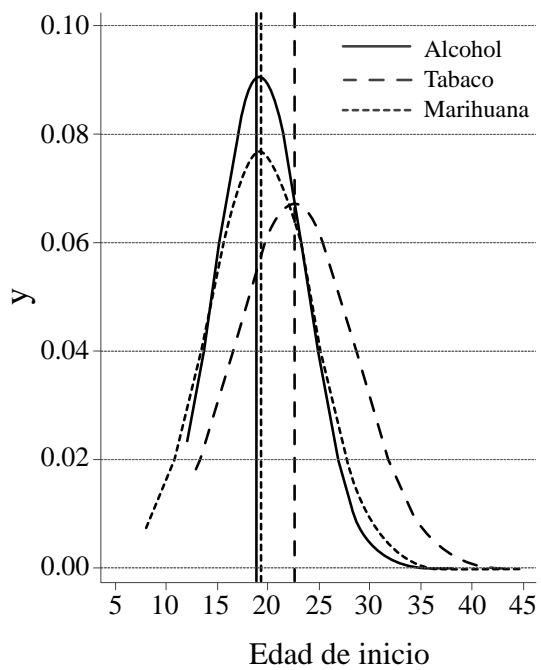

Nota: Las líneas verticales paralelas al eje de ordenadas reflejan las medias de cada función de densidad según el color correspondiente.

Fuente: elaboración propia sobre la base de ENPreCoSP. 
Si se observa el consumo de drogas sociales distinguiendo entre individuos que usan y no usan marihuana, el consumo de cigarrillos es el doble en los consumidores de marihuana que en los que no usan esta sustancia $52 \%$ versus $26 \%$, respectivamente (ver tabla A1 del anexo de la versión digital). A su vez, los primeros tienen un mayor porcentaje de fumadores severos y comienzan a consumir tabaco casi dos años antes que los no consumidores de la droga ilegal. En cuanto a las bebidas alcohólicas, el 79\% las personas que usan cannabis consume alcohol en comparación con el $47 \%$ de los individuos que no consumen, inicia casi tres años antes el consumo de alcohol y el porcentaje de personas que tiene un consumo excesivo de alcohol es 20 puntos porcentuales más que el porcentaje de personas que no utilizan la droga prohibida. De nuevo, los hombres presentan valores más altos que las mujeres.

En cuanto al consumo de cocaína y otras drogas ilegales, el acceso a las sustancias psicoactivas y el estado de salud de los consumidores de marihuana, el $26 \%$ de estos ha consumido cocaína y el $8 \%$ probó éxtasis. A su vez, el $78 \%$ de los individuos tiene conocidos que consumen alguna sustancia y un $80 \%$ tiene curiosidad por probar drogas ilegales. En cuanto al acceso a las drogas, el $79 \%$ declara tener un fácil acceso a la marihuana, el $54 \%$ declara poder conseguir fácilmente cocaína, y el $37 \%$ puede acceder al éxtasis.

Por otro lado, el 50\% de los consumidores de marihuana percibe tener una muy buena salud, $15 \%$ visitó algún médico por problemas de depresión o similar, el $24 \%$ consumió más de lo pensado y el $23 \%$ lo hizo como vía de escape. El $14 \%$ incrementó el consumo de marihuana para lograr el mismo efecto y el $6 \%$ advierte que el consumo de marihuana afectó su salud.

Dentro de los consumidores de marihuana, el 50\% ha consumido cocaína más de lo pensado en alguna ocasión, un 37\% lo hizo como vía de escape y el 30\% incrementó su uso para lograr el mismo efecto. Respecto al éxtasis, el 9\% consumió en exceso en alguna ocasión, el 1\% lo hizo como vía de escape, el 12\% tuvo que incrementar su uso para lograr el mismo efecto y el $0.8 \%$ vio afectada su salud.

Respecto a las características socio-demográficas según los individuos sean consumidores o no de drogas ilícitas, entre los consumidores hay un mayor porcentaje de hombres jóvenes, hay un menor porcentaje de casados, una mayor tasa de ocupados. Cabe destacar que entre los consumidores de cocaína el $83 \%$ son hombres mientras que en el caso de marihuana este valor es $67 \%$ y en el caso de éxtasis es $73 \%$. En cuanto al consumo según nivel educativo, hay una mayor pro- 
porción de consumidores de marihuana con nivel educativo alto con relación a los consumidores de cocaína y éxtasis que tienen un nivel educativo más bajo (tabla A3 del anexo de la versión digital).

\section{METODOLOGÍA}

En el trabajo se testea el escalonamiento en el consumo de drogas en Argentina, es decir, se estudia si el consumo de drogas legales y su edad de inicio afectan la probabilidad de consumir alguna droga ilegal de inicio, como la marihuana, y si este consumo puede llevar hacia el consumo de drogas ilegales más nocivas, tales como la cocaína y el éxtasis. El abordaje empírico se realiza sobre la base de la siguiente especificación:

$$
\operatorname{Pr}(Y=1 \mid X)_{i}=\alpha+\beta_{1} \times \text { EdadInicio }_{i}+\beta_{2} \times \text { Consumo }_{i}+X_{i}^{\prime} \theta+\mu_{i}
$$

en donde $\operatorname{Pr}(Y=1 \mid X)_{i}$ es la probabilidad de consumir una droga ilegal para el individuo $i$, EdadInicio $_{i}$ es la edad de inicio de consumo de una droga legal o ilegal de inicio, Consumo $_{i}$ es una variable binaria que vale 1 si el individuo $i$ consume la droga legal o ilegal de inicio en la actualidad, $X_{i}$ es un vector de variables de control del individuo $i$ y $\mu_{i}$ es el término de error del modelo.

Puntualmente, en $X_{i}$ se incluye información de la persona respecto al sexo, la edad, si está casado, si está ocupado, el nivel educativo, la percepción de riesgo del consumo de la droga ilegal, la condición de precariedad de la vivienda, si tiene o no cobertura de salud y la región geopolítica. En una especificación adicional, se estiman las regresiones considerando distintas variables de propensión y acceso (si ha tenido o no tratamiento médico por depresión u otros motivos, si tuvo curiosidad por probar y si tiene facilidad para conseguir la sustancia).

Se realizan dos tipos de estimaciones en forma separada. En primer lugar, se estudian los efectos del consumo de drogas legales y su edad de inicio sobre la marihuana. En cuanto a las drogas legales, se consideran tres alternativas: 1) consumo de tabaco; 2) consumo de alcohol y 3) consumo de tabaco y/o alcohol. En segundo lugar, se analizan los efectos del consumo de marihuana y su edad de inicio sobre el consumo de cocaína y éxtasis.

Si se cumple la hipótesis de puerta de entrada y escalonamiento es esperable que la edad de inicio tenga una relación negativa con la variable dependiente 
$\left(\beta_{1}<0\right)$, es decir, que las personas que empiezan a consumir a más temprana edad alguna droga legal (o ilegal de inicio) tienen una mayor propensión a consumir marihuana (o una droga ilegal más nociva), ceteris paribus. A su vez, es de esperar que el signo de $\beta_{2}$ sea positivo, lo que implica una relación positiva entre las personas que consumen la droga legal (o ilegal de inicio) y la probabilidad de usar marihuana (o una droga ilegal más nociva).

La ecuación 1 puede modelarse mediante un Probit y los coeficientes de la regresión pueden estimarse con el método de máxima verosimilitud. Sin embargo, al incluir entre los controles la edad en la que se inició el consumo de la droga legal o ilegal, solo se observa la variable dependiente para las personas que han consumido alguna vez, es decir, aquellas que tienen valor en la edad de inicio. De esta manera, $Y$ se comporta como una variable latente. En consecuencia, se emplea un modelo de selección a lo Heckman (1979), en donde la ecuación de selección mide la probabilidad de haber consumido la droga legal o ilegal de inicio y es la siguiente:

$$
\operatorname{Pr}(Z=1 \mid X)_{i}=\gamma+X_{i}^{\prime} \delta+\epsilon_{i}
$$

donde $\operatorname{Pr}(Z=1 \mid X)_{i}$ es la probabilidad del individuo $i$ de haber fumado o consumido alcohol o consumido marihuana alguna vez, $X_{i}$ es un vector con características del individuo $i$ (sexo, edad, si está casado, si está ocupado, nivel educativo, percepción de riesgo del consumo de tabaco, alcohol o marihuana y región geopolítica) $\mathrm{y}_{i}$ es el término de error del modelo.

Cabe notar que, aun incluyendo en las distintas especificaciones de la ecuación 1 variables de propensión y accesibilidad, pueden existir variables endógenas omitidas que afecten los resultados. Entre ellas pueden citarse variables biológicas, psicológicas, sociales y de contexto que no han sido relevadas en la encuesta utilizada ${ }^{6}$.

Dada la imposibilidad de controlar por el total de heterogeneidad no observada, los coeficientes encontrados no pueden interpretarse como efectos causales sino como correlaciones.

6 Tagliazucchi, et al. (2017) es un instrumento idóneo para profundizar sobre estos aspectos. 


\section{RESULTADOS}

La tabla 3 es un resumen de todas las especificaciones de la ecuación 1 estimadas cuando la variable dependiente es una variable dummy que toma valor $1 \mathrm{si}$ alguna vez probó marihuana (columnas 1.1 a 1.3) o cuando la variable dependiente es una dummy que toma valor 1 si es consumidor actual de marihuana, esto es si consumió marihuana en los últimos 30 días (columnas 2.1 a 2.3). En la tabla 4 se presentan los respectivos efectos marginales.

En todas las especificaciones, los coeficientes de edad de inicio de consumo de tabaco son negativos. Esto indicaría una relación inversa, un consumo tardío de tabaco se asociaría con una menor probabilidad de probar alguna vez o consumir marihuana, ceteris paribus. También se encuentra que los coeficientes de edad de inicio de consumo de alcohol son negativos. En cuanto a la condición de consumidor de drogas legales, ya sea tabaco y/o alcohol, los resultados sugieren una asociación directa tanto con la probabilidad de haber probado alguna vez marihuana como con la probabilidad de ser consumidor de la misma.

En cuanto a los efectos marginales encontrados para las primeras dos especificaciones (columnas 1.1 y 1.2), los mismos muestran que comenzar a fumar cigarrillos (consumir alcohol) un año antes se asocia a un aumento en la probabilidad de probar marihuana, entre 1.2 y 1.6 (1.3 y 2.1) puntos porcentuales (p. p.), ceteris paribus ${ }^{7}$. Sin embargo, una vez que se controla por acceso y propensión, se atenúan dichos efectos marginales, los que podrían pasar a ser económicamente irrelevantes. Si esto fuera así, estos últimos resultados sugerirían que la hipótesis de puerta de entrada no estaría operando.

A su vez, los efectos marginales del modelo de probabilidad de consumo de marihuana en los últimos 30 días (columnas 2.1, 2.2 y 2.3 de la tabla 4) son todos más pequeños respecto a los del modelo de probar alguna vez. Particularmente, al controlar por acceso y propensión, se obtienen efectos marginales nulos.

Los efectos marginales encontrados para las tres especificaciones anteriores podrían sugerir que el potencial efecto escalonamiento consiste en un escalonamiento en consumos ocasionales, pero no sostenidos en el tiempo. De esta manera, la iniciación en el consumo de drogas induciría a probar otras drogas, pero no a

7 En esta sección se utiliza el término "se asocia" para referir a una correlación y no a efectos causales. Los efectos marginales se analizan considerando una probabilidad entre 0 y 100. 
sostener dicho consumo en el tiempo. Esta hipótesis podría ser factible en el caso de drogas que no generen una rápida y fuerte dependencia.

Respecto a los efectos de las covariables sobre la probabilidad de consumir marihuana, los resultados son, en general, robustos a las distintas especificaciones (ver tablas A4 y A5 del anexo de la versión digital). La probabilidad de consumir marihuana es mayor para los hombres, las personas ocupadas y que no consideran que exista un riesgo alto en el consumo de la sustancia. A su vez, estar casado, tener mayor edad y tener una cobertura de salud disminuye la probabilidad de usar la sustancia. Las variables de propensión y de acceso tienen un efecto significativo sobre la probabilidad de consumir marihuana: tener algún tipo de problema como depresión, curiosidad por probar y fácil acceso a la droga se relaciona positivamente con su uso.

También se opta por explorar si el consumo de una droga blanda, como lo es la marihuana, tiene alguna relación con la probabilidad de consumo de otras drogas, como cocaína y éxtasis. La tabla 5 presenta las estimaciones de un potencial segundo escalonamiento. Los resultados sugieren una relación inversa entre la edad de inicio en el consumo de marihuana y la probabilidad de probar cocaína y éxtasis, mientras que se encuentra una relación positiva entre el consumo de marihuana y la probabilidad de consumir las otras dos sustancias psicoactivas consideradas.

La tabla 6 presenta los respectivos efectos marginales. El inicio en el consumo de marihuana un año antes respecto a los demás se asocia con un incremento de la probabilidad de consumir cocaína, entre 1.1 y 2.1 p. p. La condición de consumidor de marihuana se asocia con un incremento de la probabilidad de consumir cocaína, entre un 1.6 p. p. y 3.2 p. p. Además, estos efectos marginales se mantienen en la tercera especificación, mostrando que el inicio en el consumo marihuana un año antes se asocia con un incremento en la probabilidad de consumir cocaína de 1.8 p. p. y la condición de consumidor actual de marihuana se asocia a un aumento de 2.97 p. p.

En el caso del uso de éxtasis los resultados no son concluyentes. Si bien se encuentra una relación negativa entre la edad de inicio de consumo de marihuana y la probabilidad de probar éxtasis, así como también una relación directa entre la condición de consumidor de marihuana y la probabilidad de consumo de éxtasis, los respectivos efectos marginales cuando se controla por otros factores no son estadísticamente y económicamente significativos. En este caso las hipótesis de propensión y acceso tampoco tienen un rol importante. No obstante, las estimaciones pueden verse afectadas por el bajo número de casos positivos, lo que implica poca variabilidad de la variable dependiente. 


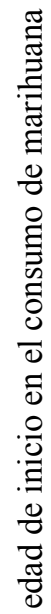

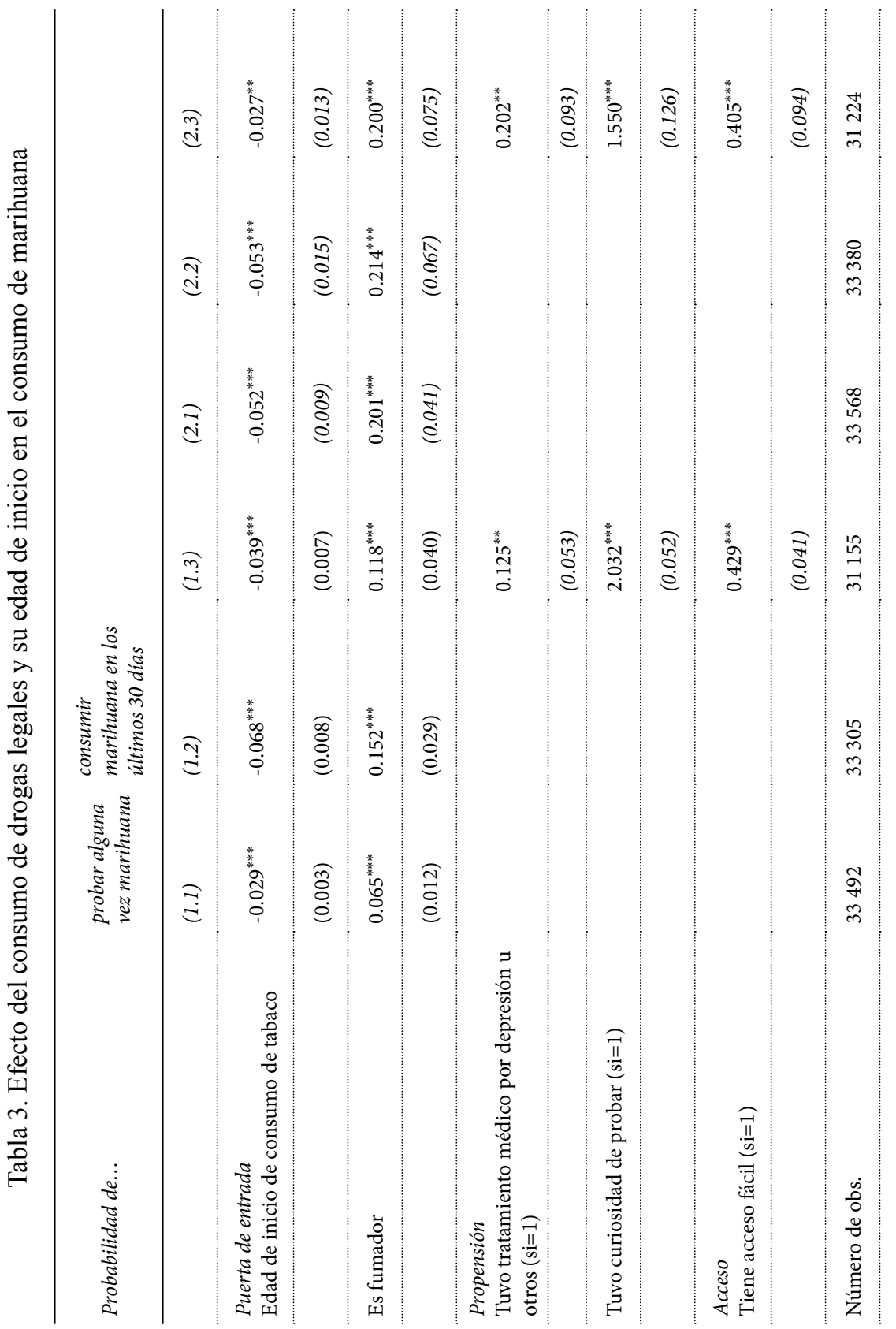




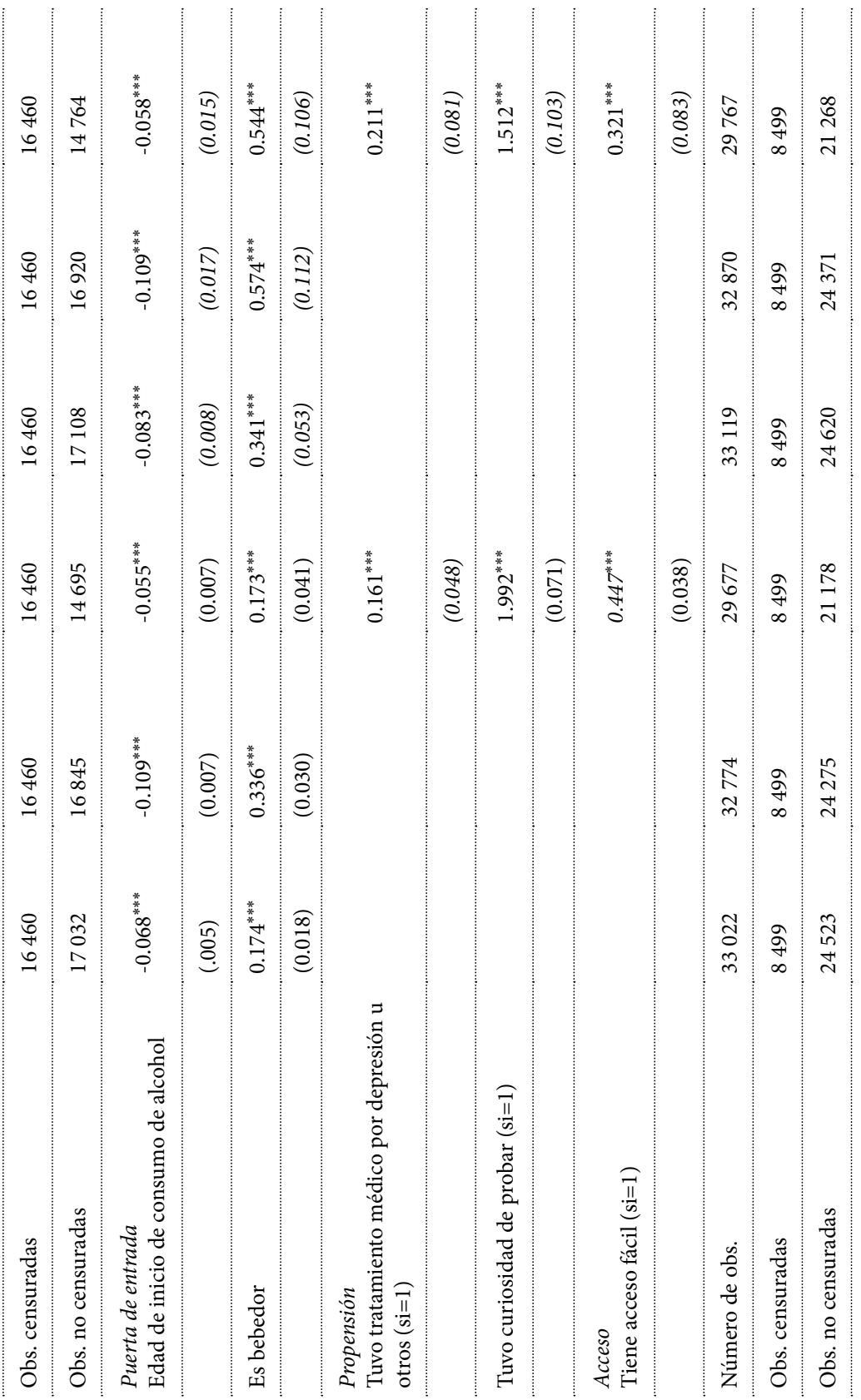




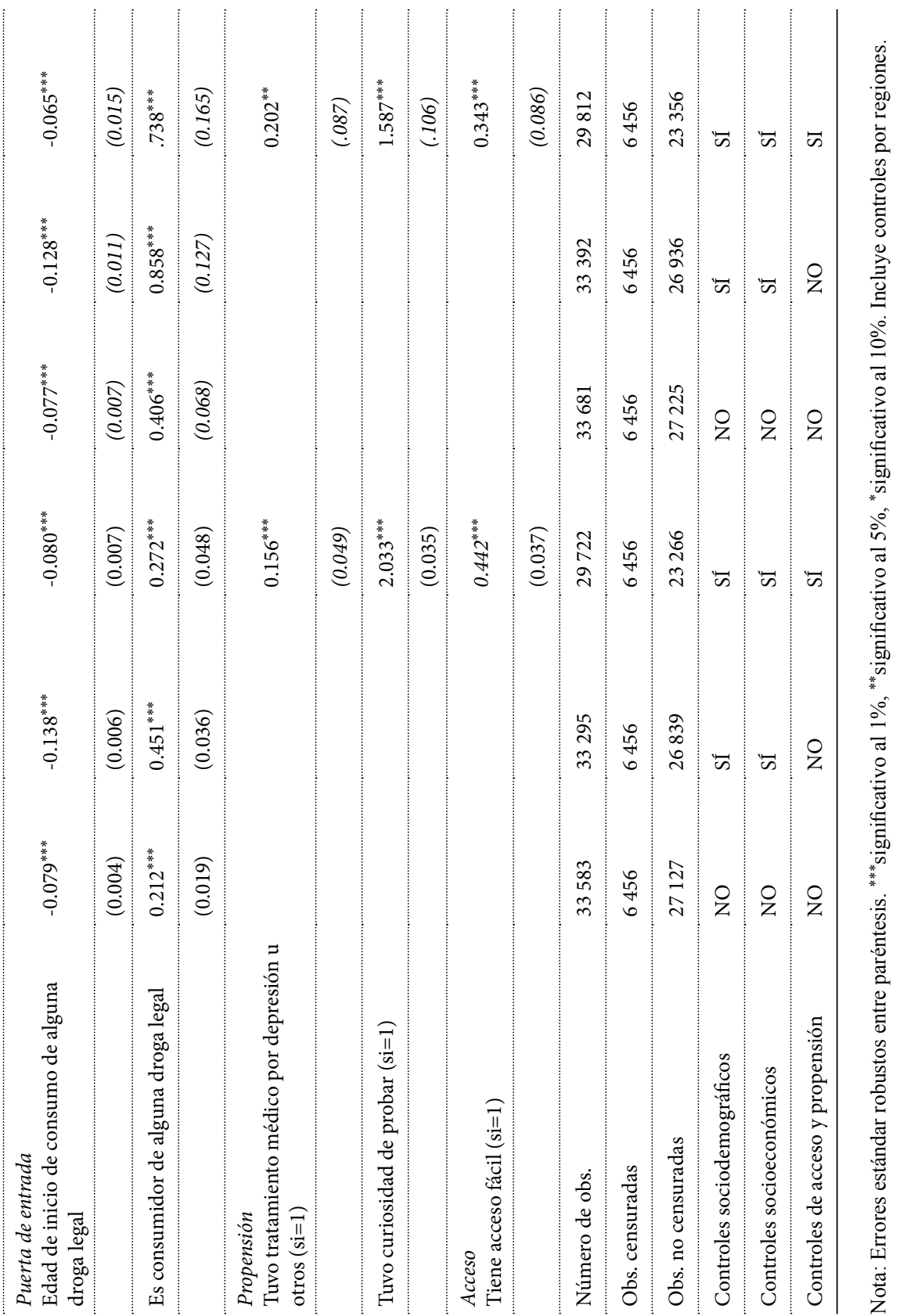


Tabla 4. Efectos marginales del consumo de drogas legales y su edad de inicio en el consumo de marihuana

$\begin{array}{ccc} & \text { probar } & \text { consumir } \\ \text { Probabilidad de ... } & \text { alguna vez } & \text { marihuana en los } \\ & \text { marihuana } & \text { últimos 30 días }\end{array}$

\begin{tabular}{|c|c|c|c|c|c|c|}
\hline & (1.1) & (1.2) & (1.3) & (2.1) & $(2.2)$ & (2.3) \\
\hline \multirow[t]{2}{*}{$\begin{array}{l}\text { Puerta de entrada } \\
\text { Edad de inicio de } \\
\text { consumo de tabaco }\end{array}$} & $-0.012^{* * *}$ & $-0.016^{* * *}$ & -0.003 & $-0.012^{* * *}$ & $-0.007^{*}$ & -0.000 \\
\hline & $(0.001)$ & $(0.003)$ & $(0.003)$ & $(0.002)$ & $(0.004)$ & $(0.000)$ \\
\hline \multirow[t]{2}{*}{ Es fumador } & $0.026^{* * *}$ & $0.037^{* * *}$ & 0.010 & $0.046^{* * *}$ & $0.027^{*}$ & 0.000 \\
\hline & $(0.005)$ & $(0.008)$ & (0.008) & $(0.008)$ & $(0.016)$ & $(0.000)$ \\
\hline
\end{tabular}

Propensión

Tuvo tratamiento médico por depresión

$\mathrm{u}$ otros $(\mathrm{si}=1)$

(0.009)

$(0.000)$

Tuvo curiosidad de $\operatorname{probar}(\mathrm{si}=1)$
0.180
$0.001^{* *}$

$(0.130)$

$(0.001)$

Acceso

$\begin{array}{lcc}\text { Tiene acceso fácil } & 0.038 & 0.000^{*}\end{array}$

$(\mathrm{si}=1)$

\begin{tabular}{|c|c|c|c|c|c|c|}
\hline & & & $(0.028)$ & & & $(0.000)$ \\
\hline Número de obs. & 17032 & 16845 & 14695 & 17108 & 16920 & 14764 \\
\hline \multirow{3}{*}{$\begin{array}{l}\text { Puerta de entrada } \\
\text { Edad de inicio de } \\
\text { consumo de alcohol }\end{array}$} & & & & & & \\
\hline & $-.021^{* * *}$ & $-.013^{* * *}$ & $-.002^{* * *}$ & $-.010^{* * *}$ & -.002 & $-.000^{* *}$ \\
\hline & $(0.001)$ & $(0.001)$ & $(0.000)$ & $(0.001)$ & $(0.002)$ & $(0.000)$ \\
\hline \multirow[t]{2}{*}{ Es bebedor } & $0.053^{* * *}$ & $0.041^{* * *}$ & $0.007^{* * *}$ & $0.043^{* * *}$ & 0.012 & $0.000^{* *}$ \\
\hline & (0.005) & $(0.005)$ & (0.002) & $(0.005)$ & (0.010) & $(0.000)$ \\
\hline \multicolumn{7}{|l|}{ Propensión } \\
\hline \multirow[t]{2}{*}{$\begin{array}{l}\text { Tuvo tratamiento } \\
\text { médico por depresión } \\
\mathrm{u} \text { otros }(\mathrm{si}=1)\end{array}$} & & & $0.007^{* * *}$ & & & $0.000^{*}$ \\
\hline & & & $(0.002)$ & & & $(0.000)$ \\
\hline
\end{tabular}




\begin{tabular}{|c|c|c|c|c|c|c|}
\hline \multirow[t]{2}{*}{$\begin{array}{l}\text { Tuvo curiosidad de } \\
\text { probar }(\mathrm{si}=1)\end{array}$} & & \multicolumn{4}{|c|}{$0.086^{* * *}$} & \multirow{2}{*}{$\begin{array}{l}0.001^{* * *} \\
(0.000) \\
0.00\end{array}$} \\
\hline & & & $(0.010)$ & & & \\
\hline $\begin{array}{l}\text { Acceso } \\
\text { Tiene acceso fácil } \\
(\mathrm{si}=1)\end{array}$ & & & $0.019^{* * *}$ & & & $0.000^{* *}$ \\
\hline & & & $(0.002)$ & & & $(0.000)$ \\
\hline Número de obs. & 24523 & 24275 & 21178 & 24620 & 24371 & 21268 \\
\hline \multirow[t]{2}{*}{$\begin{array}{l}\text { Puerta de entrada } \\
\text { Edad de inicio de } \\
\text { consumo de alguna } \\
\text { droga legal }\end{array}$} & $-0.022^{* * *}$ & $-0.011^{* * *}$ & $-0.004^{* * *}$ & $-0.009^{* * *}$ & $-0.000^{* * *}$ & $-0.000^{* *}$ \\
\hline & $(0.001)$ & $(0.001)$ & $(0.001)$ & $(0.001)$ & $(0.000)$ & $(0.000)$ \\
\hline \multirow[t]{2}{*}{$\begin{array}{l}\text { Es consumidor de } \\
\text { alguna droga legal }\end{array}$} & $0.059^{* * *}$ & $0.037^{* * *}$ & $0.012^{* * *}$ & $0.050^{* * *}$ & $0.003^{* * *}$ & $0.000^{* *}$ \\
\hline & $(0.005)$ & $(0.005)$ & $(0.003)$ & $(0.007)$ & $(0.001)$ & $(0.000)$ \\
\hline \multirow[t]{2}{*}{$\begin{array}{l}\text { Propensión } \\
\text { Tuvo tratamiento } \\
\text { médico por depresión } \\
\text { u otros }(\mathrm{si}=1)\end{array}$} & & & $0.007^{* * *}$ & & & 0.000 \\
\hline & & & $(0.002)$ & & & $(0.000)$ \\
\hline \multirow[t]{2}{*}{$\begin{array}{l}\text { Tuvo curiosidad de } \\
\text { probar }(s i=1)\end{array}$} & & & $0.090^{* * *}$ & & & $0.001^{* *}$ \\
\hline & & & $(0.012)$ & & & $(0.000)$ \\
\hline \multirow[t]{2}{*}{$\begin{array}{l}\text { Acceso } \\
\text { Tiene acceso fácil } \\
(\mathrm{si}=1)\end{array}$} & & & $0.020^{* * *}$ & & & $0.000^{* *}$ \\
\hline & & & $(0.003)$ & & & $(0.000)$ \\
\hline Número de obs. & 27127 & 26839 & 23266 & 27225 & 26936 & 23356 \\
\hline $\begin{array}{l}\text { Controles } \\
\text { sociodemográficos }\end{array}$ & $\mathrm{NO}$ & Sí & Sí & NO & SÍ & Sí \\
\hline $\begin{array}{l}\text { Controles } \\
\text { socioeconómicos }\end{array}$ & $\mathrm{NO}$ & Sí & SÍ & NO & SÍ & SÍ \\
\hline $\begin{array}{l}\text { Controles de acceso y } \\
\text { propensión }\end{array}$ & NO & NO & Sí & NO & $\mathrm{NO}$ & Sí \\
\hline
\end{tabular}

Nota: Errores estándar robustos entre paréntesis. ${ }^{* * *}$ significativo al $1 \%,{ }^{* *}$ significativo al $5 \%,{ }^{*}$ significativo al $10 \%$. Incluye controles por regiones. 
Tabla 5. Efecto del consumo de marihuana y su edad de inicio en el consumo de cocaína y éxtasis

\begin{tabular}{|c|c|c|c|c|c|c|}
\hline Probabilidad de ... & $\begin{array}{l}\text { probar } \\
\text { alguna vez } \\
\text { cocaína }\end{array}$ & $\begin{array}{l}\text { probar } \\
\text { alguna vez } \\
\text { éxtasis }\end{array}$ & & & & \\
\hline & (1.1) & $(1.2)$ & (1.3) & (2.1) & $(2.2)$ & (2.3) \\
\hline \multirow[t]{2}{*}{$\begin{array}{l}\text { Puerta de entrada } \\
\text { Edad de inicio de } \\
\text { consumo de marihuana }\end{array}$} & $-0.055^{* * *}$ & $-0.054^{* * *}$ & $-0.047^{* * *}$ & $-0.043^{* * *}$ & $-0.076^{* * *}$ & $-0.059^{* *}$ \\
\hline & $(0.008)$ & $(0.011)$ & $(0.012)$ & $(0.013)$ & $(0.016)$ & $(0.026)$ \\
\hline \multirow[t]{2}{*}{$\begin{array}{l}\text { Consumidor de } \\
\text { marihuana }\end{array}$} & $0.796^{* * *}$ & $0.821^{* * *}$ & $0.778^{* * *}$ & $0.678^{* * *}$ & $0.767^{* * *}$ & $0.522^{* *}$ \\
\hline & $(0.072)$ & $(0.122)$ & $(0.139)$ & $(0.092)$ & $(0.101)$ & $(0.222)$ \\
\hline \multirow[t]{2}{*}{$\begin{array}{l}\text { Propensión } \\
\text { Tuvo tratamiento médico } \\
\text { por depresión u otros } \\
(\mathrm{si}=1)\end{array}$} & & & $0.245^{* * *}$ & & & 0.085 \\
\hline & & & $(0.091)$ & & & $(0.103)$ \\
\hline \multirow[t]{2}{*}{$\begin{array}{l}\text { Tuvo curiosidad de } \\
\text { probar }(s i=1)\end{array}$} & & & $0.527^{* * *}$ & & & $0.557^{* *}$ \\
\hline & & & $(0.129)$ & & & $(0.229)$ \\
\hline \multirow[t]{2}{*}{$\begin{array}{l}\text { Acceso } \\
\text { Tiene acceso fácil }(\mathrm{si}=1)\end{array}$} & & & $0.644^{* * *}$ & & & $0.377^{* * *}$ \\
\hline & & & $(0.119)$ & & & $(0.145)$ \\
\hline $\begin{array}{l}\text { Controles } \\
\text { sociodemográficos }\end{array}$ & NO & SÍ & SÍ & NO & SÍ & SÍ \\
\hline $\begin{array}{l}\text { Controles } \\
\text { socioeconómicos }\end{array}$ & NO & SÍ & SÍ & NO & SÍ & SÍ \\
\hline $\begin{array}{l}\text { Controles de acceso y } \\
\text { propensión }\end{array}$ & NO & $\mathrm{NO}$ & SÍ & NO & NO & SÍ \\
\hline Número de obs. & 33705 & 33674 & 33445 & 33703 & 33672 & 33224 \\
\hline Obs. censuradas & 31259 & 31259 & 31259 & 31259 & 31259 & 31259 \\
\hline Obs. no censuradas & 2446 & 2415 & 2186 & 2444 & 2413 & 1965 \\
\hline
\end{tabular}

Nota: Errores estándar robustos entre paréntesis. ${ }^{* * *}$ significativo al $1 \%,{ }^{* *}$ significativo al $5 \%,{ }^{*}$ significativo al $10 \%$. Incluye controles por regiones. 
Tabla 6. Efectos marginales del consumo de marihuana y su edad de inicio en el consumo de cocaína y éxtasis

\begin{tabular}{|c|c|c|c|c|c|c|}
\hline Probabilidad de ... & $\begin{array}{l}\text { probar } \\
\text { alguna vez } \\
\text { cocaina }\end{array}$ & $\begin{array}{l}\text { probar } \\
\text { alguna } \\
\text { vez } \\
\text { éxtasis }\end{array}$ & & & & \\
\hline & (1.1) & $(1.2)$ & (1.3) & $(2.1)$ & $(2.2)$ & (2.3) \\
\hline \multirow{3}{*}{$\begin{array}{l}\text { Puerta de entrada } \\
\text { Edad de inicio de consumo de } \\
\text { marihuana }\end{array}$} & & & & & & \\
\hline & $-0.011^{* * *}$ & $-0.021^{* * *}$ & $-0.018^{* * *}$ & $-0.014^{* * *}$ & -0.004 & -0.001 \\
\hline & $(0.002)$ & $(0.003)$ & $(0.004)$ & $(0.004)$ & $(0.003)$ & $(0.001)$ \\
\hline \multirow[t]{2}{*}{ Consumidor de marihuana } & $0.160^{* * *}$ & $0.320^{* * *}$ & $0.297^{* * *}$ & $0.214^{* * *}$ & 0.039 & 0.007 \\
\hline & $(0.025)$ & $(0.033)$ & $(0.063)$ & $(0.029)$ & $(0.033)$ & $(0.007)$ \\
\hline \multirow{2}{*}{$\begin{array}{l}\text { Propensión } \\
\text { Tuvo tratamiento médico por } \\
\text { depresión u otros }(\mathrm{si}=1)\end{array}$} & & & $0.093^{* * *}$ & & & 0.001 \\
\hline & & & $(0.035)$ & & & $(0.002)$ \\
\hline \multirow[t]{2}{*}{ Tuvo curiosidad de probar $(\mathrm{si}=1)$} & & & $0.201^{* * *}$ & & & 0.007 \\
\hline & & & $(0.052)$ & & & $(0.007)$ \\
\hline \multirow{2}{*}{$\begin{array}{l}\text { Acceso } \\
\text { Tiene acceso fácil }(\mathrm{si}=1)\end{array}$} & & & $0.246^{* * *}$ & & & 0.005 \\
\hline & & & $(0.048)$ & & & $(0.005)$ \\
\hline Controles sociodemográficos & NO & SÍ & SÍ & NO & SÍ & SÍ \\
\hline Controles socioeconómicos & NO & SÍ & SÍ & NO & SÍ & SÍ \\
\hline $\begin{array}{l}\text { Controles de acceso y } \\
\text { propensión }\end{array}$ & NO & NO & Sí & NO & NO & SÍ \\
\hline Número de obs. & 2446 & 2415 & 2186 & 2444 & 2413 & 1965 \\
\hline
\end{tabular}

Nota: Errores estándar robustos entre paréntesis. ${ }^{* * *}$ significativo al $1 \%,{ }^{* *}$ significativo al $5 \%,{ }^{*}$ significativo al $10 \%$. Incluye controles por regiones. 
Las estimaciones de las dos etapas de Heckman para consumo de cocaína y éxtasis, en general, los signos de los coeficientes de las covariables se mantienen en las distintas especificaciones en el caso de cocaína (tablas A6 y A7 del anexo de la versión digital). La probabilidad de probar cocaína es mayor para los hombres de mayor edad, ceteris paribus. A su vez, tener mayor educación y una cobertura de salud disminuye la probabilidad de usar la sustancia. Vivir en una vivienda precaria aumenta la probabilidad, aunque su efecto no es estadísticamente significativo. Considerar que el riesgo en el consumo de cocaína es bajo o medio también aumenta la probabilidad, aunque no significativamente. Las variables de propensión y de acceso tienen un efecto significativo sobre la probabilidad de consumir cocaína: tener depresión, curiosidad por probar y fácil acceso a la droga incrementa su uso, ceteris paribus.

En el caso de éxtasis, la probabilidad de probar dicha sustancia aumenta para los hombres, a mayor educación y si considera que el riesgo de consumo es bajo. El efecto de estar casado es negativo, sugiriendo que manteniéndose todo lo demás constante, el tener una pareja disminuye la probabilidad de probar éxtasis. En cuanto a las variables de propensión y acceso, tener curiosidad de probar y acceso fácil incrementa la probabilidad de consumo de la sustancia, ceteris paribus.

Para estudiar la relación entre el consumo de droga legal y de su edad de inicio con el consumo de marihuana y la relación del consumo de marihuana y su edad de inicio con el consumo de cocaína o éxtasis, respectivamente, se estiman dos especificaciones alternativas (tablas A8 y A9 del anexo de la versión digital). La primera especificación propone un modelo lineal de probabilidad en donde la variable dependiente considera si la persona consume la droga en función de una variable que indica si el individuo ha consumido alguna vez la sustancia anterior y otra variable de interacción entre haber consumido la droga anterior con la edad de inicio en su consumo. La segunda especificación también propone un modelo lineal de probabilidad considerando la misma variable dependiente, pero en función de una variable indicadora si el individuo consume o no la droga previa actualmente, el tiempo que lleva consumiendo dicha droga (estimada como la edad menos la edad de inicio en el consumo) y la interacción entre ambas. En ambos casos se incorporan variables de controles sociodemográficas, socioeconómicas y de acceso y propensión. Las regresiones fueron estimadas por mínimos cuadrados ordinarios y los resultados encontrados van en líneas con los modelos propuestos en las tablas 3 a 6. 


\section{IMPLICANCIAS DE POLÍTICA}

Desde el punto de vista de quién diseña políticas antidrogas, es importante entender el impacto que los programas para reducir el consumo de una droga tienen sobre el consumo, presente y futuro, de esa droga y otras sustancias psicoactivas. Por un lado, reducir el consumo de una droga que es considerada puerta de entrada podría reducir el consumo de esa sustancia y de otras. Pero, por otro lado, los consumidores podrían sustituir la droga cuyo consumo se intenta reducir por otra. Así, las recomendaciones de política antidroga son diferentes dependiendo de si existe progresión en el consumo de drogas o si el consumo de las diferentes sustancias psicoactivas responde a características individuales no observadas.

Si el consumo de alcohol y tabaco genera la necesidad psicológica o fisiológica de experimentar con marihuana y esta implica una progresión hacia otras drogas, o si el consumo de las primeras reduce los costos de empezar con drogas ilegales y escalonar hacia drogas más nocivas, o si el hecho de consumir previamente drogas sociales incrementa la utilidad de consumir sustancias ilícitas, entonces los esfuerzos por prevenir o restringir el consumo de alcohol y tabaco tendrán efectos positivos sobre el consumo de otras drogas (Bretteville-Jensen y Jacobi, 2008). Pero si los consumidores no perciben de forma obvia los efectos perjudiciales para la salud que genera el consumo de alcohol y tabaco, entonces para debilitar los mecanismos de transición debería proveerse información distintiva relativa a los riesgos para la salud asociados al consumo de cada tipo de sustancias (Bretteville-Jensen et al., 2005). Si, por el contrario, el patrón secuencial de iniciación al consumo observado es consecuencia de diferencias en la accesibilidad, variar el acceso a diferentes sustancias en diferentes etapas de ciclo de vida podría bastar para modificar el comportamiento observado.

No obstante, dados los resultados de este trabajo y de otros trabajos como los ya mencionados, las dos hipótesis podrían estar actuando simultáneamente. En consecuencia, las políticas de salud pública antidrogas por el lado de la demanda deberían abarcar ambos frentes. En este sentido, Degenhardt et al. (2010) sugieren dirigir los esfuerzos de prevención hacia el consumo de todo tipo de drogas en lugar de centrarse en patrones específicos de iniciación o en el consumo de determinadas drogas para evitar la progresión en el consumo, sobre todo en jóvenes por ser quienes enfrentan el riesgo mayor de desarrollar adicciones dado su entorno y vulnerabilidad. 


\section{CONCLUSIONES}

En esta investigación se estudia la relación que tiene el consumo de drogas legales y la edad de inicio de estos hábitos sobre el consumo de marihuana y sobre la progresión hacia el consumo de drogas ilícitas más nocivas, como la cocaína y el éxtasis, en Argentina. Un aporte del trabajo, consiste en brindar evidencia descriptiva relevante para entender el fenómeno bajo estudio.

Los resultados obtenidos sugieren que el consumo de drogas legales se relaciona positivamente con la probabilidad de consumir cannabis; además, esta probabilidad se relaciona inversamente con la edad de inicio de consumo de alcohol y tabaco. A su vez, se observa una asociación positiva entre el consumo de marihuana y la probabilidad de probar cocaína y éxtasis. Como en el caso anterior, se obtiene una relación negativa entre la edad de inicio de marihuana y la probabilidad de probar estas dos sustancias.

No obstante, cuando se incorporan en las especificaciones variables de accesibilidad y propensión, se atenúan los efectos. En algunos casos los mismos hasta podrían ser nulos y no resultar económicamente relevantes. De esto surge la discusión de si el potencial efecto escalonamiento puede consistir en un escalonamiento en consumos ocasionales, pero no sostenidos en el tiempo, pudiendo tener implicancias sumamente importantes para las políticas de consumo de drogas.

Los resultados presentados aquí pretenden ser una primera aproximación a este tipo de comportamiento de consumidores. La presencia de heterogeneidad no observada y la dificultad de encontrar estrategias empíricas idóneas hacen que no sea posible interpretar los resultados como efectos causales. La agenda futura busca avanzar en esta dirección. Esta línea de investigación implica varios desafíos relacionados con la información disponible y con el rol de la oferta de drogas (narcotráfico) para ahondar en el acceso a drogas ilegales.

\section{REFERENCIAS BIBLIOGRÁFICAS}

Aaron Ginzler, J., Cochran, B. N., Domenech-Rodriguez, M., Mari Cauce, A., \& Whitbeck, L. B. (2003). Sequential progression of substance use among homeless youth: an empirical investigation of the gateway theory. Substance Use \& Misuse, 38 (3-6), 725-758. Recuperado de doi.org/10.1081/ JA-120017391. 
Badiani, A., Boden, J. M., De Pirro, S., Fergusson, D. M., Horwood, L. J., \& Harold, G. T. (2015). Tobacco smoking and cannabis use in a longitudinal birth cohort: evidence of reciprocal causal relationships. Drug and alcohol dependence, 150, 69-76. Recuperado de doi.org/10.1016/j.drugalcdep.2015.02.015.

Becker, G. S., Grossman, M., \& Murphy, K. (1991). Rational addiction and the effect of price on consumption. American Economic Review, 81 (2), 237241. Recuperado de http://www.jstor.org/stable/2006861.

Becker, G. S., \& Murphy, K. M. (1988). A theory of rational addiction. Journal of Political Economy, 96 (4), 675-700. Recuperado de https://EconPapers. repec.org/RePEc:ucp:jpolec:v:96:y:1988:i:4:p:675-700.

Beenstock, M., \& Rahav, G. (2002). Testing gateway theory: do cigarette prices affect illicit drug use? Journal of Health Economics, 21, 679-698. Recuperado de doi.org/10.1016/S0167-6296(02)00009-7.

Bretteville-Jensen, A. L., \& Jacobi, L. (2008). Climbing the drug staircase: A bayesian analysis of the initiation of hard drug use. IZA Discussion Paper No. 3879. Recuperado de https://www.econstor.eu/bitstream/10419/35531/1/588006742.pdf

Bretteville-Jensen, A. L., Melberg, H. O., \& Jones, A. M. (2005). Sequential patterns of drug use initiation - can we belive in the gateway theory. HEDG Working Paper No. 05/09. Recuperado de https://www.york.ac.uk/media/ economics/documents/herc/wp/05_09.pdf

Chaloupka, F. J. (1991). Rational addictive behavoir and cigarette smoking. Journal of Political Economy, 99 (4), 722-742. Recuperado de doi. org/10.1086/261776.

Degenhardt, L., Chiu, W., Conway, K., Dierker, L., Glantz, M., Kalaydjian, A.,..., $\&$ Kessler, R. (2009). Does the 'gateway'matter? associations between the order of drug use initiation and the development of drug dependence in the national comorbidity study replication. Psychological medicine, 39 (1), 157167. Recuperado de doi.org/10.1017/S0033291708003425.

Degenhardt, L., Dierker, L., Chiu, W. T., Medina-Mora, M. E., Neumark, Y., Sampson, N., Alonso, J.,..., \& Kessler, R. C. (2010). Evaluating the drug use "gateway" theory using cross-national data: Consistency and associations of the order of initiation of drug use among participants in the who world mental health surveys. Drug and Alcohol Dependence, 108, 84-97. Recuperado de doi.org/10.1016/j.drugalcdep.2009.12.001.

Dembo, R., Williams, L., Wothke, W., \& Schmeidler, J. (1994). The relationships among family problems, friends' troubled behavior, and high risk youths' alcohol/other drug use and delinquent behavior: A longitudinal study. Inter- 
national Journal of the Addictions, 29 (11), 1419-1442. Recuperado de doi. org/10.3109/10826089409048717.

DeSimone, J. (1998). Is marijuana a gateway drug? Eastern Economic Journal, 24 (2), 149-164. Recuperado de https://EconPapers.repec.org/RePEc:eej:eeconj:v:24:y:1998:i:2:p:149-164.

Deza, M. (2015). Is there a stepping stone effect in drug use? separating state dependence from unobserved heterogeneity within and between illicit drugs. Journal of Econometrics, 184 (1), 193-207. Recuperado de doi. org/10.1016/j.jeconom.2014.08.005.

Fairman, B. J., Furr-Holden, C. D., \& Johnson, R. M. (2019). When marijuana is used before cigarettes or alcohol: demographic predictors and associations with heavy use, cannabis use disorder, and other drug-related outcomes. Prevention Science, 20 (2), 225-233. Recuperado de doi.org/10.1007/ s11121-018-0908-3.

Farrelly, M. C., Bray, J. W., Zarkin, G. A., \& Wendling, B. W. (2001). The joint demand for cigarettes and marijuana: evidence from the national household surveys on drug abuse. Journal of Health Economics, 20 (1), 51-68. Recuperado de doi.org/10.1016/S0167-6296(00)00067-9.

Fergusson, D. M., Boden, J. M., \& Horwood, L. J. (2006). Cannabis use and other illicit drug use: testing the cannabis gateway hypothesis. Addiction, 101 (4), 556-569. Recuperado de doi.org/10.1111/j.1360-0443.2005.01322.x.

Gómez, R. A. (2013). La legislación penal argentina sobre drogas. Una aproximación histórica. V Congreso Internacional de Investigación y Práctica Profesional en Psicología, Universidad de Buenos Aires, pp. 83-88. Recuperado de https://www.aacademica.org/000-054/292

Goldstein, P. J. (1985). The drugs/violence nexus: A tripartite conceptual framework. Journal of Drug Issues, 15 (4), 493-506. Recuperado de doi. org/10.1177/002204268501500406.

Golub, A., \& Johnson, B. D. (1994). The shifting importance of alcohol and marijuana as gateway substances among serious drug abusers. Journal of studies on alcohol, 55 (5), 607-614. Recuperado de doi.org/10.15288/ jsa.1994.55.607.

González, P. (2017). ¿Cómo medimos los daños causados por las drogas? En E. Tagliazucchi, M. Sigman, L. Cancela, A. Rieznik, J. C. Godoy, J. C. Mansilla \& C. Damín. Un libro sobre drogas. Recuperado de https://elgatoylacaja.com.ar/sobredrogas/como-medimos-los-danos-causados-por-las-drogas/ Heckman, J. J. (1979). Sample selection bias as a specification error. Econometrica, 47 (1), 153-161. Recuperado de http://www.jstor.org/stable/1912352. 
Kandel, D. B. (1975). Stages in adolescent involvement in drug use. Science 190, 912-924. Recuperado de https://science.sciencemag.org/content/190/4217/912.full.pdf

MacCoun, R. J., \& Reuter, P. (2001). Drug War Heresies: Learning from Other Vices, Times, and Places. New York: Cambridge University Press.

Mackesy-Amiti, M. E., Fendrich, M., \& Goldstein, P. J. (1997). Sequence of drug use among serious drug users: typical vs atypical progression. Drug and alcohol dependence, 45 (3),185-196. Recuperado de doi.org/10.1016/ S0376-8716(97)00032-X.

Mansilla, J. C. (2017). Nacimiento y crisis del Prohibicionismo. En E. Tagliazucchi, M. Sigman, L. Cancela, A. Rieznik, J. C. Godoy, J. C. Mansilla \& C. Damín. Un libro sobre drogas. Recuperado de https://elgatoylacaja.com.ar/ sobredrogas/como-medimos-los-danos-causados-por-las-drogas/

Melberg, H. O., Jones, A. M., \& Bretteville-Jensen, A. L. (2010). Is cannabis a gateway to hard drugs? Empirical Economics, 38 (3), 583-603. Recuperado de doi.org/10.1007/s00181-009-0280-z.

Nutt, D. (2012). Drugs - Without the Hot Air: Minimising the Harms of Legal and Illegal Drugs. Cambridge: UIT Cambridge Ldt.

Nutt, D., King, L. A., Saulsbury, W., \& Blakemore, C. (2007). Development of a rational scale to assess the harm of drugs of potential misuse. The Lancet, 369, 1047-1053. Recuperado de doi.org/10.1016/S0140-6736(07)60464-4.

OEA (2013). El problema de drogas en las Américas. Alternativas legales y regulatorias. Technical report, Organización de los Estados Americanos. Recuperado de http://www.cicad.oas.org/drogas/elinforme/informeDrogas2013/ alternativasLegales_ESP.pdf

OEA (2019). Informe sobre el consumo de drogas en las Américas. Technical report, Organización de los Estados Americanos. Recuperado de http:// cicad.oas.org/Main/ssMain/HTML\%20REPORT\%20DRUG\%202019/ mobile/index.html

Otten, R., Mun, C. J., \& Dishion, T. J. (2017). The social exigencies of the gateway progression to the use of illicit drugs from adolescence into adulthood. Addictive Behaviors, 73, 144-150. Recuperado de doi.org/10.1016/j.addbeh.2017.05.011.

Pacula, R. L. (1997). Letter: Economic modelling of the gateway effect. Health Economics, 6 (5), 521-524. Recuperado de doi.org/10.1002/(SICI)10991050(199709)6:5<521::AID-HEC301>3.0.CO;2-6.

Pacula, R. L. (1998a). Adolescent alcohol and marijuana consumption: is there a gateway effect? NBER, Working Paper No. 6348, 1-46. Recuperado de https://www.nber.org/papers/w6348 
Pacula, R. L. (1998b). Does increasing the beer tax reduce marijuana consumption? Journal of Health Economics, 17 (5), 557-585. Recuperado de doi. org/10.1016/S0167-6296(97)00039-8.

Parker, H., \& Bottomley, T. (1996). Crack cocaine and drugs: Crime careers. Manchester: SPARC, Department of Social Policy and Social Work, University of Manchester.

Pautassi, R. (2017). Bases neurofisiológicas de la adicción. En E. Tagliazucchi, M. Sigman, L. Cancela, A. Rieznik, J. C. Godoy, J. C. Mansilla \& C. Damín. Un libro sobre drogas. Recuperado de https://elgatoylacaja.com.ar/sobredrogas/ como-medimos-los-danos-causados-por-las-drogas/

Powell, M. A. (2011). A comprehensive analysis of the drug-crime relationship. Research Papers, Paper No. 100. Recuperado de https://opensiuc.lib.siu. edu/gs_rp/100/

Pudney, S. (2003). The road to ruin? sequences of initiation to drugs and crime in britain. The Economic Journal, 113 (486), 182-198. Recuperado de doi. org/10.1111/1468-0297.00107.

Richmond-Rakerd, L. S., Slutske, W. S., \& Wood, P. K. (2017). Age initiation and substance use progression: A multivariate latent growth analysis. Psychology of Addictive Behaviors, 31 (6), 664-675. Recuperado de doi. org/10.1037/adb0000304.

Saffer, H. \& Chaloupka, F. (1999). The demand for illicit drugs. Economic Inquiry, 37 (3), 401-411. Recuperado de doi.org/10.1111/j.1465-7295.1999. tb01439.x.

Samper-Pizano, E. (2017). UNGASS 2016 y el nuevo paradigma sobre el problema mundial de las drogas. Desafios, 29, 353-367. Recuperado de http://www.scielo.org.co/scielo.php?script=sci_arttext\&pid=S0124-40352017000200353

Sartor, C., Agrawal, A., Lynskey, M., Duncan, A., Grant, J., Nelson, E., Madden, P., Heath, A., \& Bucholz, K. (2013). Cannabis or alcohol first? differences by ethnicity and in risk for rapid progression to cannabis-related problems in women. Psychological medicine, 43 (4), 813-823. Recuperado de doi. org/10.1017/S0033291712001493.

Sartor, C. E., Hipwell, A. E., \& Chung, T. (2019). Alcohol or marijuana first? correlates and associations with frequency of use at age 17 among black and white girls. Journal of studies on alcohol and drugs, 80 (1), 120-128. Recuperado de doi.org/10.15288/jsad.2019.80.120.

Sedronar (2017). Estudio nacional en población de 12 a 65 años sobre consumo de sustancias psicoactivas, argentina. Factores de riesgo en el consumo de sustancias psicoactivas. Technical report, Sedronar. Recuperado de http://www. observatorio.gov.ar/media/k2/attachments/2017-10-05ZEncuestaZHogaresZconZcuestionario.pdf 
Sedronar (2018). Boletín estadístico del perfil de pacientes asistidos, transferencias por subsidios a tratamiento y línea de atención 141. I trimestre de 2018. Technical Report, Sedronar. Recuperado de http://observatorio.gob.ar/ media/k2/attachments/boletinZprimerZtrimestreZ2018ZcorregidoZ19-Z6. pdf

Stevens, A., Trace, M., \& Bewley-Taylor, D. (2005). Reducing drug related crime: An overview of the global evidence. Beckley Fundation, Report No. 5. Recuperado de http://altgeorgia.ge/documents/publikaciebi\%20ENG/BeckleyFoundation_Report_05.pdf

Tagliazucchi, E., Sigman, M., Cancela, L., Rieznik, A., Godoy, J. C. Mansilla, J. C. \& C. Damín. Un libro sobre drogas. Recuperado de https://elgatoylacaja. com.ar/sobredrogas/como-medimos-los-danos-causados-por-las-drogas/

UNGASS (2016). Documento final de periodo extraordinario de sesiones de la Asamblea General de las Naciones Unidas sobre el problema mundial de las drogas celebrado en 2016. Nueva York: UNDOC. Recuperado de https:// www.unodc.org/documents/postungass2016/outcome/V1603304-S.pdf

van Ours, J. C. (2003). Is cannabis a stepping-stone for cocaine? Journal of Health Economics, 22 (4), 539-554. DOI: 10.1016 / S0167-6296 (03) 00005-5

White, H. R., \& Gorman, D. M. (2000). Dynamics of the drug-crime relationship. Criminal Justice, 1 (15), 151-218. Recuperado de https://pdfs.semanticscholar.org/631c/da5cdbb7a28f58b556a348436bcad534fd37.pdf? $\mathrm{ga}=2.57967978 .1764127859 .1566219323-1925571626.1566219323$.

(C) 2019 por los autores; licencia otorgada a la revista Estudios económicos. Este artículo es de acceso abierto y distribuido bajo los términos y condiciones de una licencia Atribución-No Comercial 3.0 Unported (CC BY-NC 3.0) de Creative Commons. Para ver una copia de esta licencia, visite http://creativecommons.org/ licenses/by-nc/3.0/ 